Special issue of the International Conference on Computational and Experimental Science and Engineering (ICCESEN 2014)

\title{
Superconducting Cavity Utilization for Linear Accelerator Systems
}

\author{
H.D. YILDIZ ${ }^{a, *}$, R. CAKIR ${ }^{b}$, D. PORSUK ${ }^{c}$ \\ ${ }^{a}$ Institute of Accelerator Technology, Ankara University, Ankara, Turkey \\ ${ }^{b}$ Nanotechnology Department, RTE University, Rize, Turkey \\ ${ }^{c}$ Physics Department, Dumlupinar University, Kutahya, Turkey
}

\begin{abstract}
Self amplified stimulated emission free electron laser production from electron energy above $1.5 \mathrm{GeV}$ is studied to obtain 1-100 nm laser wavelength. Design simulations of linear accelerating system with gun and enjector system has been done in more detail by considering space charge effect for electron beam. Electromagnetic field for superconducting cavities, solenoid fields, cavity defects such as Higher Order Modes, Multipacting, Lorentz Force Detuning, and beam behaviour along the beamline are very important details for this study. Optimised cavity cell design layout are provided in order to obtain quality factor $10^{9}$ for designed cavities throughout the main linear acceleration. To determine coupling factor, and zero modes and also other modes with relative errors are searched.
\end{abstract}

DOI: 10.12693/APhysPolA.128.B-246

PACS: 29.20.Ej, 42.60.By

\section{Introduction}

Self amplified stimulated emission free electron laser (SASE-FEL) 1-100 nm range based on electron beam with energy up to $3 \mathrm{GeV}$ is studied. SASE-FEL has unique properties such as high peak power, high peak brilliance $\left(\approx 10^{30}\right.$ foton $/\left(\mathrm{s} \mathrm{mm}^{2} \operatorname{mrad}^{2} \% 0.1 \mathrm{bg}\right)$, spatial coherency, high average brilliance $\left(\approx 10^{21}\right.$ photon/(s.mrad $\left.{ }^{2} \% 0.1 \mathrm{bg}\right)$, fast wavelength tunability (X-rays-UV) and timing, monochromatic, and subpicosecond pulse length. In this study, at $1300 \mathrm{MHz}$ frequency, electrons are extracted from the $\mathrm{Cs}_{2} \mathrm{Te}$ photocathode by driving laser (Nd:YLF). These electrons can be accelerated by RF inside the superconducting cavities. Figure 1 shows gun cavity, main solenoid, injector cavities and quadrupoles.

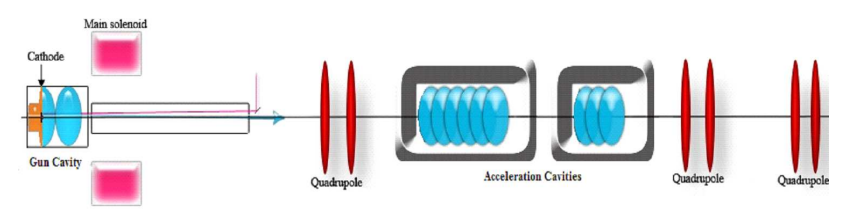

Fig. 1. SASE FEL injector system consist of a cathode, $1 \frac{1}{2}$-cell superconducting gun cavity, main solenoid, preaccelerator cavities and many quadrupoles.

Beam is accelerated with an $23 \mathrm{MV} / \mathrm{m}$ acceleration gradient inside the gun cavity. Transverse, longitudinal emittance beam size is studied and tried to optimize by varying main solenoid field strength and placement. We need to get optimized beam properties at the gun and then diagnose at some point to obtain high quality beam

*corresponding author; e-mail: hdyildiz@ankara.edu.tr at the entrance of the undulator. Optimized beam for the injector and gun properties are presented in Table I.

TABLE I

Optimized beam and RF gun component properties.

\begin{tabular}{l|c}
\hline \hline Parameter and unit & Value \\
\hline Solenoid field $[\mathrm{T}]$ & 0.1868 \\
Gradient $[\mathrm{MV} / \mathrm{m}]$ & 23 \\
Peak current, $I_{\text {peak }}[\mathrm{kA}]$ & 2 \\
Electron beam energy, $E_{\text {beam }}[\mathrm{MeV}]$ & 25 \\
Bunch charge, $Q[\mathrm{nC}]$ & 0.1 \\
Normalized emittance, $\varepsilon_{N}[\pi \mathrm{mm}$ mrad] & $\sim 2.6$ \\
Frequency [GHz] & 1.3 \\
Gun UHV $[\mathrm{mbar}]$ & $10^{-10}-10^{-11}$
\end{tabular}

\section{Cavity design}

Elliptical cavity shape is considered in the simulations to accelerate the beam up to speed of light. To design $1 \frac{1}{2}$-cell gun cavity, we used Poisson/Superfish code [1]. The shape of the cavity can be firstly drawn into two dimensions. Each cavity has a bigger ellipse on the equator and a smaller ellipse on the iris area. Both ellipses are connected with a tangent line in Fig. 2. To define the cavity shape, there must be at least 7 parameters: horizontal and vertical axis of both ellipses $A, B$ and $a, b$, $R_{\text {equ }}, R_{\text {iris }}$, and $L$. Since the frequency, and so the wavelength, is given, the cavity length $L$ is fixed. Big values of $R_{\text {iris }}$ causes high cell-to-cell coupling but it becomes hard to optimize the geometry thus we consider the same $R_{\text {iris }}$ value during the optimization. Superfish/Poisson varies the $R_{\text {equ }}$ value for given parameters to get the desired frequency so there is only 4 parameters left to change; $A, B, a, b$. However, because of the ease of calculations, instead of four parameters, program optimizes $A, A / B, a / b$ and $\alpha$, which is the wall inclination angle. Optimized geometry values are given in Table II. 
TABLE II

Optimized design geometry values for $1 \frac{1}{2}$ and higher number of cells.

\begin{tabular}{l|c|c}
\hline \hline Parameters & inner $[\mathrm{cm}]$ & end $[\mathrm{cm}]$ \\
\hline Length of half cell $L / 2$ & \multicolumn{2}{|c}{5.71347} \\
Iris radius $R_{\text {iris }}$ & 3.5 & 3.5 \\
Equator radius $R_{\text {eq }}$ & \multicolumn{2}{|c}{10.2298} \\
Horizontal axes of the iris elipse $A$ & 4.6622 & 3.73239 \\
Vertical axes of the iris ellipse $B$ & 4.57078 & 3.6592 \\
Iris ellipse axises ratio $A / B$ & \multicolumn{2}{|c}{1.02} \\
Horizontal axes of the equator & 0.986747 & 1.95352 \\
elipse $a$ & 1.40964 & 2.79075 \\
Vertical axes of the equator & \multicolumn{2}{|c}{$\mid$} \\
ellipse $b$ & \multicolumn{2}{|c}{0.7} \\
Equator ellipse axises ratio $a / b$ & 7 \\
Wall angle $\alpha$ [deg] & \multicolumn{2}{|c}{7}
\end{tabular}

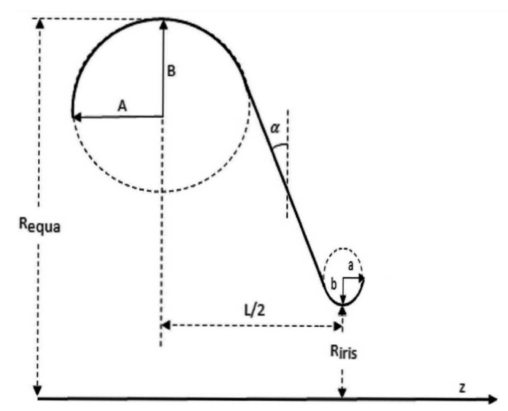

Fig. 2. Half of a cavity cell design geometry.

Gun cavity is simulated both Poisson/Superfish and CST program [2]. Figure 3 shows $1 \frac{1}{2}$-cell gun cavity field lines for $\pi$ mode while Fig. 4 shows $1 \frac{1}{2}$-cell cavity in 3 dimesions.

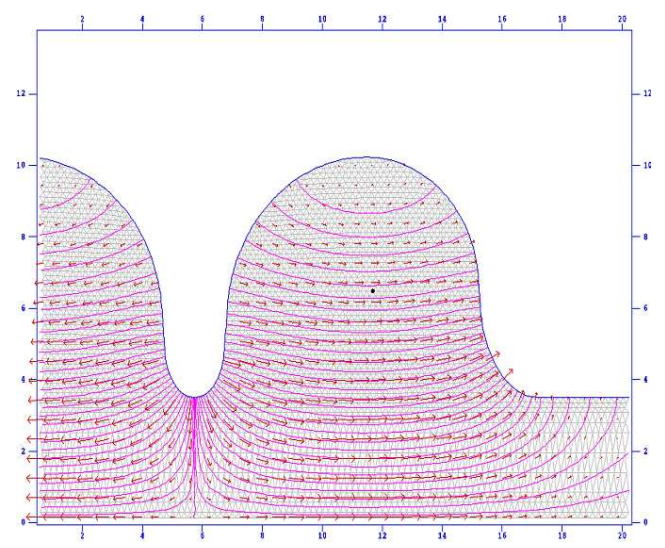

Fig. 3. $1 \frac{1}{2}$-cell gun cavity field lines for $\pi$ mode.

The quality factor $Q$ is the most important parameter that characterises RF loses in the cavity. The quality factor $Q$ of an RF cavity is defined as

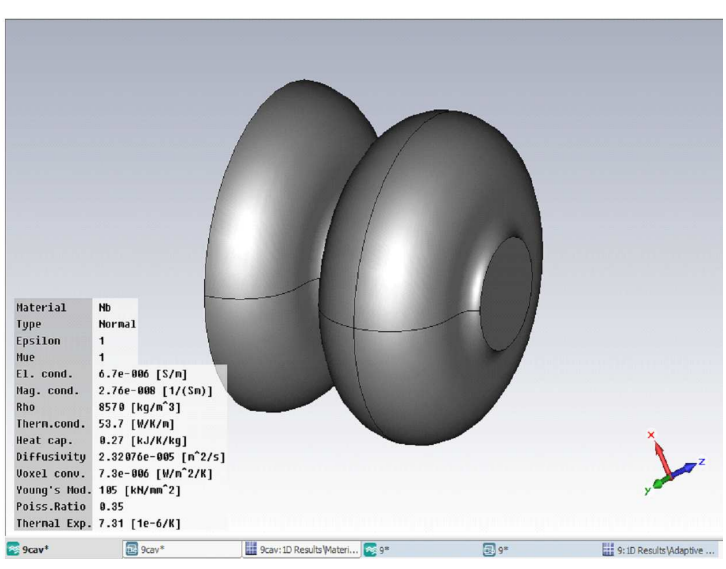

Fig. 4. $\quad 1 \frac{1}{2}$-cell cavity in 3 dimesions.

$$
Q=\frac{\omega U}{P},
$$

where $\omega=2 \pi f$ is the angular frequency, $U$ is the cavity stored energy, and $P$ is the power dissipated in the walls per radian of the $\mathrm{RF}$ cycle.

Beam behaviour along the beamline is simulated by utilizing ASTRA [3]. Particles are traced by ASTRA. Figure 5 shows beam behaviour at the exit of the injector for transverse phase space and front, and side view of the particle beams.
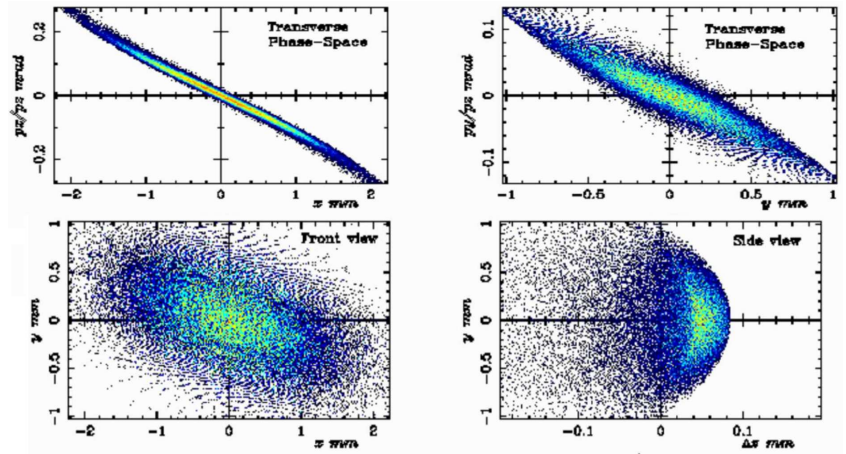

Fig. 5. Distribution of the beam at the transverse phase space and front, and side view of the particles distribution respectively along the beamline. $z=5.2 \mathrm{~m}$.

\section{Conclusion}

Cavity is designed and optimized properties of the beam are obtained. Low transverse emittance $(\leq 2.6)$, beam size and beam energy $(\sim 25 \mathrm{MeV})$ at the end of the injector are met with our expectations.

\section{References}

[1] http://laacg.lanl.gov/laacg/services/ download_sf.phtml.

[2] CST Microwave Studio, CST GMbH, Darmstadt.

[3] K. Flöttmann, Astra homepage. 